\title{
Vasculitis con necrosis digital asociada con interferón beta
}

\author{
Evangelina Miretti, Laura Encinas, Marcela Colazo, María Haye Salinas, Verónica Saurit, Francisco Caeiro, \\ Alejandro Alvarellos \\ Hospital Privado Centro Médico de Córdoba. Carrera de Posgrado de Reumatología, Universidad Católica de Córdoba.
}

\section{Resumen}

El Interferón (IFN) es una citoquina producida por distintas poblaciones de células y, a partir de su estructura, se han desarrollado varias drogas biológicas utilizadas en un amplio espectro de enfermedades. Se han descripto lesiones cutáneas y casos de síndrome de Raynaud severo con gangrena digital con IFN alfa y beta.

En este reporte presentamos una paciente con necrosis digital asociada a la administración de IFN beta.

\section{Introducción}

El interferón (IFN) es una citoquina producida por células inmunocompetentes en respuesta a diversos estímulos. Se identifican cinco tipos de IFN: alfa, beta, gama, tau y omega ${ }^{1}$.

Numerosas drogas biológicas con una estructura similar se han sintetizado, entre otras el IFN beta, utilizado en el tratamiento de mieloma, leucemia mieloide crónica, hepatitis crónica, esclerosis sistémica, síndrome hipereosinofílico, psoriasis, esclerosis múltiple y melanoma ${ }^{2}$.

Los eventos adversos más frecuentes son: fiebre, síndrome gripal, gastrointestinales, psiquiátricos, disfunción hepática, exacerbación de psoriasis preexistente, arritmia, neumonitis y falla renal' ${ }^{2}$.

La isquemia es una complicación reconocida del IFN, principalmente IFN alfa. Los hallazgos varían desde vasoespasmo leve y transitorio, úlceras y livedo reticularis, a oclusión arterial con necrosis digital ${ }^{2}$. El síndrome de Raynaud es raro, y el tipo severo, que lleva a la isquemia y necrosis digital es excepcional ${ }^{3}$. En casos aislados se describió isquemia miocárdica, oftálmica, muscular y del sistema nervioso central. En la mayoría de los casos no se encontraron anticuerpos o los mismos no fueron de valor en el diagnóstico o seguimiento de las lesiones descriptas ${ }^{2}$.

Presentamos un caso de necrosis digital severa asocia$\mathrm{da}$ al tratamiento con IFN beta- $1 \mathrm{~b}$ en una paciente con esclerosis múltiple.

\section{Summary}

Interferon (IFN) is a cytokine produced by different immune competent cells and, based on its structure, several IFN biologic drugs and are now used as treatment for a wide spectrum of diseases. There are reports of cutaneous side effects and cases of severe Raynaud's syndrome with digital gangrene especially with both alpha and beta IFN. We report a patient who developed digital necrosis associated with beta Interferon.

\section{Caso clínico}

Mujer de 36 años con antecedente de esclerosis múltiple de un año y medio de evolución, tratada con IFN beta- $1 \mathrm{~b} 44$ mg subcutáneo 3 veces por semana por seis meses.

Consulta por hipoestesia de la última falange del 3er dedo de la mano izquierda de 3 semanas de evolución seguido de cianosis y úlcera periungueal muy dolorosa (Figuras 1 y 2). También presentaba lesiones induradas tipo hematoma alrededor del ombligo, en las zonas de inyección del IFN.

El resto del examen: corazón y pulsos normales sin otras lesiones vasculíticas o tromboembólicas.

Se le realizó ecografía doppler de miembros superiores sin signos de obstrucción al flujo; ecocardiograma y capilaroscopia, los cuales fueron normales.

Anticuerpos antinucleares con patrón nucleolar, título: 1/5120. Factor reumatoideo, anticardiolipinas, anticoagulante lúpico, beta 2-microglobulina, anti Ro, La, Sm, RNP, anti Scl 70 y ANCA negativos. Complemento, tests de coagulación, proteinograma, PCR y VSG normales.

Se suspende el IFN beta-1b subcutáneo, pero la lesión evolucionó a necrosis en el pulpejo al mes (Figuras 3 y 4). Inició pulsos de ciclofosfamida de $750 \mathrm{mg}$ endovenoso/ mes y prednisona $40 \mathrm{mg} /$ día vía oral a dosis decrecientes con rápida mejoría a los 3 meses y resolución completa posterior (Figuras 4 y 5). Cambia el tratamiento para su

\section{Correspondencia}

Naciones Unidas 346 - X5016KEH, Córdoba

E-mail: vangemiretti@yahoo.com.ar 


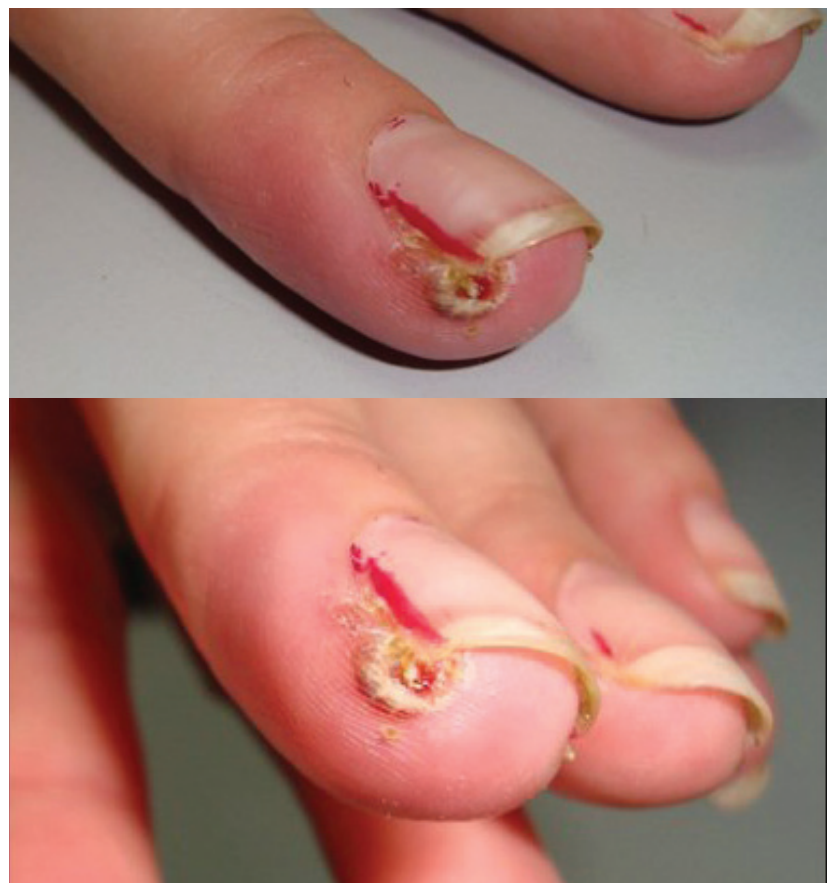

Figuras 1 y 2. Lesión al momento de la presentación.

esclerosis múltiple por otra droga, sin nueva reactivación de la vasculitis.

\section{Discusión}

La esclerosis múltiple es una patología neurológica crónica caracterizada por inflamación de la sustancia blanca y desmielinización y un curso clínico alternante entre recaídas y remisiones ${ }^{4}$. El tratamiento con interferón (IFN) beta natural intratecal y la administración subcutánea de IFN beta- $1 \mathrm{~b}$ recombinante ha demostrado disminuir la frecuencia de las exacerbaciones ${ }^{4,5,6}$.

Las citopenias relacionadas con el IFN beta- $1 \mathrm{~b}$ en esclerosis múltiple son el efecto colateral más frecuente y dependientes de la dosis.

Hay pocos reportes de vasculitis asociada a IFN beta y principalmente corresponden a vasculitis sistémicas de pequeños vasos con manifestaciones cutáneas generalizadas que responden tras el retiro del IFN con resolución inmediata ${ }^{7}$. Son más escasos aun los reportes de vasculitis asociada a cianosis y necrosis digital, y la mayoría de estos casos están asociados a IFN alfa para tratamiento de melanoma o hepatitis $\mathrm{C}^{1,3}$. En general, los síntomas ceden al suspender la droga. Otros casos necesitan tratamiento con vasodilatadores, anticoagulantes, antiplaquetarios, corticoesteroides y citotóxicos, llegando raramente a la amputación de los dedos necróticos².

En lo referente a los escasos reportes que relacionan al interferón beta con vasculitis y necrosis digital, E. Linden y col. en 1998 describen una mujer de 35 años con síndrome de Raynaud asociado a IFN beta- $1 \mathrm{~b}$ para esclerosis múltiple. Un año después de comenzar el tratamiento presentó palidez marcada y dolor en 3 dedos de una mano que luego adquirió coloración negruzca con cambios tróficos. Tuvo rápida mejoría al suspender IFN y anticoagulación ${ }^{8}$.

De Broucker reportó una paciente de 37 años con esclerosis múltiple tratada con interferón beta-1A 6 millones de unidades subcutáneas por un año. Consultó por síndrome de Raynaud en manos con isquemia digital severa. La paciente presentó anticuerpos antinucleares $+1 / 200$ con patrón antinucleolar, que estaban ausentes previamente y capilaroscopia normal. Los síntomas desaparecieron 3 meses después de suspender el tratamiento y de la indicación de Iloprost e.v. y anticoagulación?.

Otro caso reportado en el año 2000, una mujer de 58 años con E.M que, dos semanas después de iniciar tratamiento con IFN beta-1b, desarrolla cianosis y dolor en dedos de una mano seguido de necrosis isquémica en el pulpejo del 4to dedo, tratada con heparina, bolos de ciclofosfamida (1 gramo) mensuales y metilprednisolona (1 gramo), seguidos de $60 \mathrm{mg} /$ día de prednisona vía oral, con lo que se logra limitar los síntomas ${ }^{4}$.

En numerosos trabajos se ha reportado la presencia de autoanticuerpos como antinucleares, antimicrosomal y antitiroglobulinas entre los usuarios de IFN beta, los cuales fluctúan con el tiempo y en un $25 \%$ de los casos ya se encuentran presentes antes del tratamiento con IFN ${ }^{10}$, e incluso fueron reportados casos de lupus eritematoso sistémico (LES) inducidos por IFN beta- $1 \mathrm{~b}^{11}$.

Las reacciones en el sitio de inyección subcutánea a interferón beta-1b, como las que presentó nuestra paciente en la pared abdominal, son comunes en esclerosis múltiple. Se han descripto principalmente 5 tipos de reacciones:

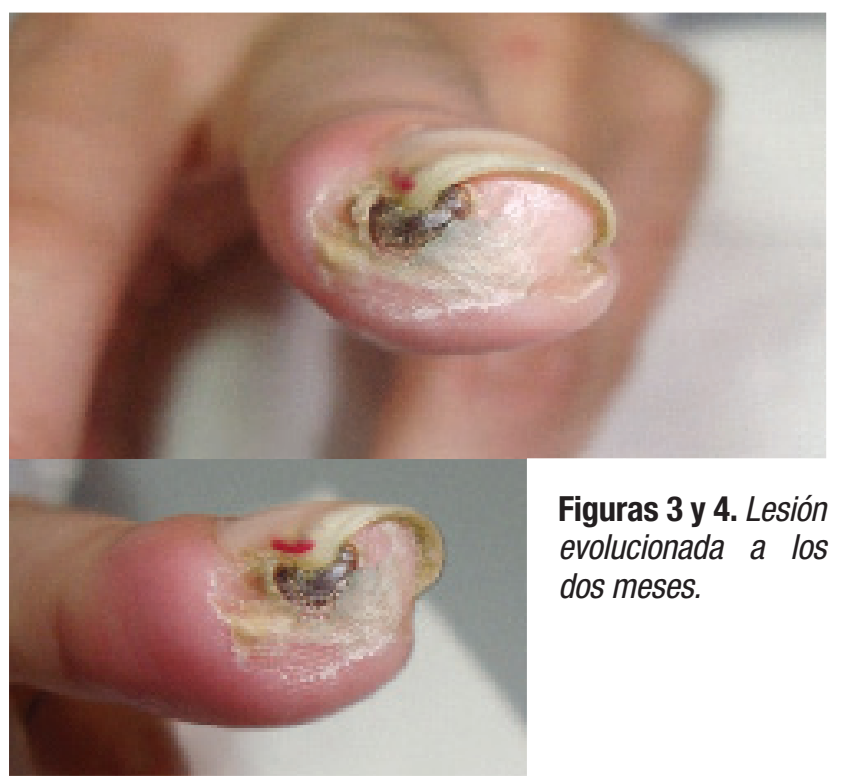




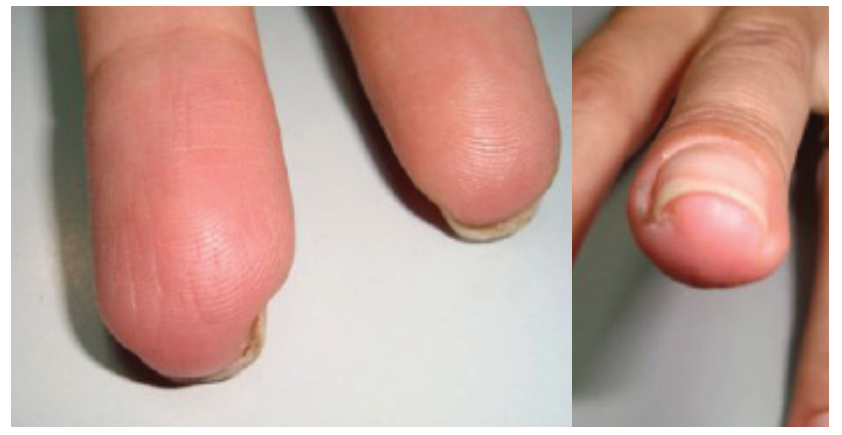

Figuras 5 y 6. Lesión curada tras el tratamiento a los 6 meses.

eritema local, hematoma, y dolor relacionado a la técnica de inyección, infección cutánea y subcutánea, atrofia subcutánea y menos frecuentemente necrosis con sobreinfección, la cual fue reportada en el 1-3\% de los pacientes ${ }^{12}$. También se han descripto placas de vasculitis linfocítica inespecífica ${ }^{13}$ o úlceras cutáneas en sitio de inyección ${ }^{14}$ y paniculitis, con lipoatrofia posterior a la misma en algunos $\operatorname{casos}^{15}$.

Reportamos el caso de una paciente con vasculitis necrotizante en un dedo de la mano asociada con tratamiento con IFN beta-1b para E.M. El cuadro se resolvió tras discontinuar la droga y tratamiento inmunosupresor con esteroides y ciclofosfamida.

Por los casos reportados previamente de síndrome de Raynaud y gangrena digital en asociación con interferón tanto alfa como beta, conociendo que ambas familias de IFN presentan modo de acción similar, y ante la falta de evidencia de signos de otra enfermedad, creemos que es válido afirmar que nuestra paciente tuvo una vasculitis necrotizante con isquemia digital asociada con la administración de IFN beta-1b.

\section{Bibliografía}

1. Al-Zahrani H, Gupta V, Minden MD, Messner HA, Lipton JH. Vascular events associated with alpha interferon therapy. Leukemia \& Lymphoma, 2003; 44(3):471-475.

2. Schapira D, Nahir AM, Hadad N. Interferon-induced Raynaud's syndrome. Semin Arthritis Rheum 2002; 32:157-162.

3. Husein-ElAhmed H, Callejas-Rubio JL, Ortega Del Olmo R, Ríos-Fernandez R, Ortego-Centeno N. Severe Raynaud syndrome induced by adjuvant interferon alfa in metastatic melanoma. Current Oncology 2010; 17(4):122-123.

4. Cruz B, Queiroz ED, Nunes SV, Cruz Filho A, Campos GB, Monteiro EL, et al. Fenomeno de Raynaud grave associado a terapia con interferon-beta para esclerose múltipla. Arq Neuropsiquiatr 2000; 58(2-B):556-559.

5. Interferon beta- $1 \mathrm{~b}$ in the treatment of multiple sclerosis. Final outcome of the randomized controlled trial. The IFNB Multiple Sclerosis Study Group and the University of British Columbia MS/MRI Analysis Group. Neurology 1995; 45:1277-1285.

6. Lawrence Jacobs, Cookfair DL, Rudick RA, Herndon RM, Richert JR, Salazar AM, et al. Intramuscular Interferon Beta-1a for disease progression in relapsing multiple sclerosis. Ann Neurol 1996; 39(3):285-294.

7. Scublinsky D, Barreña MA, Payaslián S, Correale J. Vasculitis cutánea y plaquetopenia asociadas a terapia con interferón beta-1a. Quinto caso en el mundo. Rev Arg Reumatol 2012; 23(1):49-51.

8. Linden D. Severe Raynaud's phenomenon associates with interferon-beta treatment for multiple sclerosis. Lancet 1998 Sep 12; 352:878-9.

9. De Broucker T, François Lhote. Phénomène de Raynaud sévère associé à un traitement par interféron beta-1a et fluoxétine. Ann Med Intern 2000; 151(5):424-425.

10. Speciale L, Saresella M, Caputo D, Ruzzante S, Mancuso R, Calvo MG, et al. Serum auto antibodies presence in multiple sclerosis patients treated with beta-interferon 1a and 1b. J Neurovirol. 2000 May; 6 Suppl 2:S57-61.

11. Sladkova V, Mares J, Lubenova B, Hlustik P, Kanovsky P. Drug-induced systemic lupus ery thematosus in interferon beta- $1 \mathrm{~b}$ therapy. Neuro Endocrinol Lett. 2011; 32(1):4-6.

12. Casoni F, Merelli E, Bedin R, Martella A, Cesinaro A, Bertolotto A. Necrotizing skin lesions and NABs development in a multiple sclerosis patient treated with IFN beta-1b. Multip Scler 2003; 9:420423.

13. Zoguereh D, Boucraut J, Beau-Salinas F, Bodiguel E, Lechapois D, Pomet E. Cutaneous vasculitis with renal impairment complicating interferon-beta 1 a therapy for multiple sclerosis. Rev Neurol 2004; 160(11):1081-1084.

14. Inafuku H, Kasem Khan MA, Nagata T, Nonaka S. Cutaneous ulceration following subcutaneous interferon beta injection to a patient with multiple sclerosis. J Dermatol 2004 Aug; 31(8):671-7.

15. O'Sullivan SS, Cronin EM, Sweeney BJ, Bourke JF, Fitzgibbon J. Panniculitis and lipoatrophy after subcutaneous injection of interferon beta- $1 \mathrm{~b}$ in a patient with multiple sclerosis. J Neurol Neurosurg Psychiatry 2006 Dec; 77(12):1382-3. Epub 2006 Jun 16. 